\title{
LMI-BASED ROBUST CONTROL OF UNCERTAIN NONLINEAR SYSTEMS VIA POLYTOPES OF POLYNOMIALS
}

\author{
MARCElino SÁNCHEZ ${ }^{a}$, Miguel BERNAL $^{a, *}$ \\ ${ }^{a}$ Department of Electric and Electronics Engineering \\ Sonora Institute of Technology, 5 de Febrero 818 Sur, CP 85000, Ciudad Obregon, Sonora, Mexico \\ e-mail: miguel. bernaleitson. edu.mx
}

\begin{abstract}
This investigation is concerned with robust analysis and control of uncertain nonlinear systems with parametric uncertainties. In contrast to the methodologies from the field of linear parameter varying systems, which employ convex structures of the state space representation in order to perform analysis and design, the proposed approach makes use of a polytopic form of a generalisation of the characteristic polynomial, which proves to outperform former results on the subject. Moreover, the derived conditions have the advantage of being cast as linear matrix inequalities under mild assumptions.
\end{abstract}

Keywords: robust control, uncertain systems, nonlinear systems, linear parameter-varying systems, LMIs.

\section{Introduction}

The study of linear parameter varying (LPV) systems whose uncertainty lies in a box-like compact has long ago been developed by subsuming them in the class of linear differential inclusions (Apkarian and Gahinet, 1995). Via the direct Lyapunov method, stability of the resulting polytope of linear systems can be established by checking the feasibility of a set of linear matrix inequalities (LMIs) (Boyd et al., 1994; Amato et al., 1996). LMI feasibility tests can be optimally solved (i.e., they have a global minimum) and numerical routines are available for implementation (Gahinet et al., 1995; Sturm, 1999).

Mimicking the LPV/LMI methodologies, sufficient conditions for analysis and control of nonlinear systems via exact convex quasi-LPV representations (Shamma and Cloutier, 1992) have resulted in a well-established framework (Tanaka and Wang, 2001). Improvements on the sufficiency of conditions have been threefold: Pólya-like relaxations exploiting the representation convexity (Sala and Ariño, 2007), different classes of Lyapunov functions (Guerra and Vermeiren, 2004; González and Bernal, 2016) and control laws (Baumann and Rugh, 1986; González et al., 2017), and more general models (Sala and Ario, 2009; Guerra et al., 2015).

\footnotetext{
${ }^{*}$ Corresponding author
}

Problem statement. Eigenvalue conditions such as those employed for linear systems based on the characteristic polynomial may be useful as a fourth path to tackle the aforementioned conservativeness in the quasi-LPV/LMI framework: instead of a convex state-space representation based on $\dot{x}=A(x) x$, this work explores a convex polynomial alternative based on $\operatorname{det}(s I-A(x))=0$. This approach is inspired in Kharitonov's theorem (Kharitonov, 1978), which subsumes the coefficient space of a family of polynomials within a box; this can be used to establish the stability of LPV systems with frozen parameters such as $\dot{x}(t)=(A+\Delta A) x(t)$ (Xu et al., 1993); some of these tests have been successfully expressed as LMIs (Ebihara et al., 2012).

Methodology. Uncertain nonlinear systems with frozen parameter uncertainty are considered; their stability is analyzed through a polytope of polynomials remindful of the characteristic polynomial of linear systems. LMI results are found based on the edge theorem, which establishes the stability of a convex sum of polynomials (Bartlett et al., 1988). Computational efficiency is improved by reducing the number of vertexes needed to be checked (Barber et al., 1996); conservativeness is tackled by Pólya-like relaxations. For stabilization, the methodologies of Barmish et al. (1992), Dorf and Bishop (1998) as well as Henrion et al. (2003) have been adapted to our proposal. 
Contribution. A methodology for LMI-based robust analysis and control of uncertain nonlinear systems via polytopes of polynomials is developed; it overcomes the feasibility domain of matrix-based stability (Tanaka et al., 2003; González and Bernal, 2016) and stabilization results (Rhee and Won, 2006; González et al., 2017).

Organisation. Section 2 explains how to obtain-under mild conditions - an exact polytope of polynomials representing the generalized characteristic polynomial of an uncertain nonlinear system, based on which sufficient stability conditions are developed; Section 3 provides sufficient conditions for stabilization under the same methodology avoiding the bilinear matrix inequality (BMI) nature of the problem with the aid of the uncertainty-cluster approach of Henrion et al. (2003). Examples are provided along the paper to illustrate the advantages over matrix-based techniques. Conclusions and future work are discussed in Section 4

\section{Robust stability}

Consider an affine-in-control uncertain nonlinear system

$$
\dot{x}(t)=A(x, \theta) x(t)+B(x, \theta) u(t),
$$

where $x(t) \in \mathbb{R}^{n}, u(t) \in \mathbb{R}^{m}$, and $\theta \in \mathbb{R}^{r}$ are the state, input, and uncertainty vectors, respectively; $A(\cdot, \cdot)$ : $\mathbb{R}^{n} \times \mathbb{R}^{r} \rightarrow \mathbb{R}^{n \times n}$ and $B(\cdot, \cdot): \mathbb{R}^{n} \times \mathbb{R}^{r} \rightarrow \mathbb{R}^{n \times m}$ are sufficiently smooth nonlinear matrix functions.

\section{Assumptions.}

(i) $\|A(\cdot, \cdot)\| \leq \bar{A}$ and $\|B(\cdot, \cdot)\| \leq \bar{B}$ for some finite constants $\bar{A}, \bar{B} \in \mathbb{R}$ and any matrix norm $\|\cdot\|$, within a compact set $\mathcal{C} \subseteq\left(\mathbb{R}^{n} \times \mathbb{R}^{r}\right)$ such that $(0, \theta) \in \mathcal{C}$.

(ii) Uncertainties $\theta$ are assumed to be frozen as this work intends to establish stability from a generalization of the characteristic polynomia 1 ;

(iii) Since this section is concerned with stability analysis of unforced systems, $u(t)=0$.

Rewriting a bounded nonlinear expression, irrespective of whether it depends on the state or uncertainties, is a simple task 2 To illustrate this, consider the term $\theta \in\left[\theta^{0}, \theta^{1}\right]$; then

$$
\theta=\underbrace{\left(\frac{\theta^{1}-\theta}{\theta^{1}-\theta^{0}}\right)}_{\omega_{0}(\theta)} \theta^{0}+\underbrace{\left(\frac{\theta-\theta^{0}}{\theta^{1}-\theta^{0}}\right)}_{\omega_{1}(\theta)} \theta^{1},
$$

\footnotetext{
${ }^{1}$ In general, stability of linear time-varying (LTV) systems cannot be established via an eigenvalue condition: LTV systems with a stable characteristic polynomial can have unbounded solutions for initial conditions arbitrarily close to the origin; see Example 4.22 in the work of Khalil (2002).

${ }^{2}$ See Lemma 1 in the work of Tapia et al. (2017).
}

where $\omega_{0}(\theta)+\omega_{1}(\theta)=1$. Moreover, $0 \leq \omega_{i}(\theta) \leq 1, i \in$ $\{0,1\}$ for any $\theta \in\left[\theta^{0}, \theta^{1}\right]$. Similarly, a bounded nonlinear expression of the states can be algebraically rewritten as above, for functions $\omega_{0}(\cdot)$ and $\omega_{1}(\cdot)$ dependent on the states $x$ instead of uncertainties $\theta$.

Traditionally, the quasi-LPV/LMI framework uses the direct Lyapunov method along with a convex rewriting of (1) in order to establish sufficient LMI conditions for analysis and control. The convex model is a polytope of linear systems where every nonlinearity and uncertainty has been expressed as in (2) (i.e., they are captured in so-called weighting functions such as $\omega_{0}$ and $\omega_{1}$ therein); its vertexes are linear systems whose state matrices are all the combinations of maxima and minima of the uncertainties and nonlinearities. The convex model and the original system are the same: the former is not an approximation (Taniguchi et al., 2001).

In this work, the methodology just described is employed as in Algorithm 1 to obtain-within a compact of interest $\mathcal{C}$ containing the origin-an exact polytope of polynomials for an expression remindful of the characteristic polynomial in linear contexts; we will refer to it as the generalized characteristic polynomial of (10):

$$
\begin{aligned}
p(s, x, \theta)= & \operatorname{det}(s I-A(x, \theta)) \\
= & s^{n}+a_{n-1}(x, \theta) s^{n-1}+\cdots+a_{1}(x, \theta) s \\
& +a_{0}(x, \theta) .
\end{aligned}
$$

Importantly, the algorithm below introduces conservativeness because there are several options for choosing nonlinearities and uncertainties, leading to different polytopes (modelling choices (Robles et al., 2017)). Moreover, there are different ways of using the polytope vertices for analysis and synthesis (sum relaxation choices (Sala and Ariño, 2007)).

As is well-known, the stability of a linear system can be inferred from that of its characteristic polynomial; a stable polynomial has all its roots in $\mathbb{C}^{-}$. In general, this criterium cannot be applied for nonlinear systems; nevertheless, it turns out that nonlinear systems holding the assumptions for (1) allow such an eigenvalue condition to be employed.

Theorem 1. The origin $x=0$ of the nonlinear uncertain system (1) with $u(t)=0$, having a stable generalized characteristic polynomial (3) in the compact set $\mathcal{C}$, is asymptotically stable.

Proof. Since $p(s, x, \theta)$ is stable for all $(x, \theta) \in \mathcal{C}, A(x, \theta)$ is a Hurwitz matrix for all $(x, \theta) \in \mathcal{C}$. In particular, for a fixed $\theta_{0} \in \theta \exists P \in \mathbb{R}^{n \times n}$ such that

$$
P=P^{T}>0, \quad P A\left(0, \theta_{0}\right)+A^{T}\left(0, \theta_{0}\right) P<0,
$$

defining $T_{1}=P A\left(0, \theta_{0}\right)+A^{T}\left(0, \theta_{0}\right) P, \Delta A=\left[\Delta a_{i j}\right] \in$ $\mathbb{R}^{n \times n}$ such that $\Delta a_{i j} \in[-\alpha, \alpha]$ with $\alpha>0$, and 
Algorithm 1. Polytope of polynomials.

Step 1. Identify the different terms in $a_{i}(x, \theta), i \in$ $\{0,1, \ldots, n-1\}$, which depend exclusively on the states $x$ : let us group them in $z(x)=\left[z_{1}(x) z_{2}(x) \cdots z_{p}(x)\right]^{T}$; the remaining terms are grouped in $\zeta(x, \theta)=\left[\zeta_{1}(x, \theta)\right.$ $\left.\zeta_{2}(x, \theta) \cdots \zeta_{\rho}(x, \theta)\right]^{T}$.

Step 2. For each $z_{i}(x) \in\left[z_{i}^{0}, z_{i}^{1}\right]$ and $\zeta_{j}(x, \theta) \in\left[\zeta_{j}^{0}, \zeta_{j}^{1}\right]$, write the following convex sums as described above 3

$$
\begin{gathered}
z_{i}(x)=\underbrace{\left(\frac{z_{i}^{1}-z_{i}(x)}{z_{i}^{1}-z_{i}^{0}}\right)}_{w_{0}^{i}\left(z_{i}(x)\right)} z_{i}^{0}+\underbrace{\left(\frac{z_{i}(x)-z_{i}^{0}}{z_{i}^{1}-z_{i}^{0}}\right)}_{w_{1}^{i}\left(z_{i}(x)\right)} z_{i}^{1}, \\
\zeta_{j}(x, \theta)=\underbrace{\left(\frac{\zeta_{j}^{1}-\zeta_{j}(x, \theta)}{\zeta_{j}^{1}-\zeta_{j}^{0}}\right)}_{\omega_{0}^{j}\left(\zeta_{j}(x, \theta)\right)} \zeta_{j}^{0}+\underbrace{\left(\frac{\zeta_{j}(x, \theta)-\zeta_{j}^{0}}{\zeta_{j}^{1}-\zeta_{j}^{0}}\right)}_{\omega_{1}^{j}\left(\zeta_{j}(x, \theta)\right)} \zeta_{j}^{1} .
\end{gathered}
$$

In the compact set $\mathcal{C}$, nonlinear functions $w_{k}^{i}\left(z_{i}(x)\right)$ and $\omega_{k}^{j}\left(\zeta_{j}(x, \theta)\right)$ satisfy the convex sum property, i.e., $w_{0}^{i}\left(z_{i}(x)\right)+w_{1}^{i}\left(z_{i}(x)\right)=1,0 \leq w_{k}^{i}\left(z_{i}(x)\right) \leq 1$, and $\omega_{0}^{j}\left(\zeta_{j}(x, \theta)\right)+\omega_{1}^{j}\left(\zeta_{j}(x, \theta)\right)=1,0 \leq \omega_{k}^{j}\left(\zeta_{j}(x, \theta)\right) \leq 1$.

Step 3. Considering $\mathbf{i}=\left(i_{1}, i_{2}, \ldots, i_{p}\right), \forall k: i_{k} \in$ $\{0,1\}, \mathbf{w}_{\mathbf{i}}(z)=w_{i_{1}}^{1} w_{i_{2}}^{2} \cdots w_{i_{p}}^{p}, \mathbf{j}=\left(j_{1}, j_{2}, \ldots, j_{\rho}\right)$, $\forall k: j_{k} \in\{0,1\}$, and $\omega_{\mathbf{j}}(\zeta)=\omega_{j_{1}}^{1} \omega_{j_{2}}^{2} \cdots \omega_{j_{\rho}}^{\rho}$, write (3) as the following equivalent convex sum of polynomials:

$$
p(s, x, \theta)=\sum_{\mathbf{i}} \sum_{\mathbf{j}} \mathbf{w}_{\mathbf{i}} \omega_{\mathbf{j}} p_{\mathbf{i j}}(s),
$$

where $p_{\mathbf{i j}}(s)=\left.p(s, x, \theta)\right|_{\mathbf{w}_{\mathbf{i}}=1, \omega_{\mathbf{j}}=1}$ are the vertex polynomials of the desired polytope, $\mathbf{i j}$ being the $(p+$ $\rho)$-uple which results from $\mathbf{i}$ and $\mathbf{j}$ put together.

$T_{2}=P \Delta A+\Delta A^{T} P$, we can always select $\alpha>0$ such that $\lambda_{\max }\left(T_{2}\right)<-\lambda_{\max }\left(T_{1}\right)$, where $\lambda_{\max }$ stands for the maximum eigenvalue of the argument. Then, since any pair of symmetric matrices $(B, C)$ satisfies $\lambda_{\max }(B+C) \leq \lambda_{\max }(B)+\lambda_{\max }(C)$, it follows that $T_{1}+T_{2}=P\left(A\left(0, \theta_{0}\right)+\Delta A\right)+\left(A\left(0, \theta_{0}\right)+\Delta A\right)^{T} P<0$. Thus, since there is a sufficiently small compact set $\mathcal{C}_{0}$ such that the set of matrices $\left\{A\left(x, \theta_{0}\right): x \in \mathcal{C}_{0}\right\}$ is a subset of the family $A\left(0, \theta_{0}\right)+\Delta A$, we obtain

$$
P A\left(x, \theta_{0}\right)+A^{T}\left(x, \theta_{0}\right) P<0, \quad \forall x \in \mathcal{C}_{0},
$$

which implies the existence of a quadratic Lyapunov function $V(x)=x^{T} P x$ that establishes the asymptotic stability of the origin of $\dot{x}=A\left(x, \theta_{0}\right) x$ as a consequence of $p\left(s, x, \theta_{0}\right)$ being stable. Since the argument above can be repeated for each $\theta \in \mathcal{C}$, the proof is completed.

The theorem above reduces to a linearization argument if there are no uncertainties, since $A(0)$ corresponds to the state matrix of the linearized model; it does not provide a way of estimating the domain of attraction nor establishing the stability of $p(s, x, \theta)$ for all $(x, \theta) \in \mathcal{C}$ : the former requires point wise analysis (Johansen, 2000; Kwiatkowski et al., 2006), which is out of the scope of this report, while the latter will be solved through a polytopic representation (6)

Stability of polytopes of polynomials has been studied via the Hurwitz matrix; for a given polynomial $p(s)=a_{n} s^{n}+a_{n-1} s^{n-1}+\cdots a_{1} s+a_{0}$, it is defined as

$$
H(p(s))=\left[\begin{array}{ccccccc}
a_{n-1} & a_{n-3} & a_{n-5} & \cdots & 0 & 0 & 0 \\
a_{n} & a_{n-2} & a_{n-4} & \cdots & \vdots & \vdots & \vdots \\
0 & a_{n-1} & a_{n-3} & \cdots & \vdots & \vdots & \vdots \\
\vdots & a_{n} & a_{n-2} & \ddots & 0 & \vdots & \vdots \\
\vdots & 0 & a_{n-1} & \ddots & a_{0} & \vdots & \vdots \\
\vdots & \vdots & a_{n} & \ddots & a_{1} & 0 & \vdots \\
\vdots & \vdots & 0 & \cdots & a_{2} & a_{0} & \vdots \\
\vdots & \vdots & \vdots & \cdots & a_{3} & a_{1} & 0 \\
0 & 0 & 0 & \cdots & a_{4} & a_{2} & a_{0}
\end{array}\right]
$$

The next result is an LMI test for the stability of a convex combination of two polynomials; it is based on the results of Białas (1985) and the LMI test found in the work of Ebihara et al. (2012):

Lemma 1. (Sanchez and Bernal, 2017) A convex combination of two $n$-degree stable polynomials $p_{1}(s)=$ $s^{n}+a_{n-1} s^{n-1}+\cdots+a_{1} s+a_{0}$ and $p_{2}(s)=s^{n}+$ $b_{n-1} s^{n-1}+\cdots+b_{1} s+b_{0}$

$$
\lambda p_{1}(s)+(1-\lambda) p_{2}(s), \quad \lambda \in[0,1],
$$

is stable if and only if there exists $M \in \mathbb{R}^{n \times n}$, such that $M+M^{T}>0$ and $H_{12} M+M^{T} H_{12}^{T}<0$ with $H_{12}=$ $-H\left(p_{1}(s)\right) H^{-1}\left(p_{2}(s)\right)$.

Similarly, convex combinations of an arbitrary finite number of polynomials have been developed by Bartlett et al. (1988) via the edge theorem, which states that it suffices to check the exposed edges of a polytope; thus, the LMI test that follows establishes stability of $p(s, x, \theta)$ for all $(x, \theta) \in \mathcal{C}$.

Theorem 2. The origin $x=0$ of the nonlinear uncertain system (1) with $u(t)=0$, having a generalized characteristic polynomial (3) with the exact convex representation (6) in the compact set $\mathcal{C}$, is asymptotically stable if there exist matrices $M_{\mathrm{ijkl}} \in \mathbb{R}^{n \times n}$, such that the following LMIs are feasible:

$$
M_{\mathrm{ijkl}}+M_{\mathrm{ijkl}}^{T}>0, \quad H_{\mathrm{ijkl}} M_{\mathrm{ijkl}}+M_{\mathrm{ijkl}}^{T} H_{\mathrm{ijkl}}^{T}<0,
$$

with $H_{\mathrm{ijkl}}=-H\left(p_{\mathbf{i j}}(s)\right) H^{-1}\left(p_{\mathbf{k l}}(s)\right), p_{\mathbf{i j}}$ stable polynomials, $\mathbf{i}, \mathbf{k} \in\{0,1\}^{p}, \mathbf{j}, \mathbf{l} \in\{0,1\}^{\rho}, \mathbf{i j} \neq \mathbf{k} \mathbf{l}$. 
Proof. The conditions (9) are equivalent to those in Lemma 1 for each combination of vertexes in the polytope, which, by the edge theorem (Bartlett et al., 1988) guarantees that the whole polytope is stable, which in turn implies asymptotic stability of the origin by Theorem 1 .

The above result works on the assumption that the polynomial (6) has a constant degree for all $(x, \theta) \in \mathcal{C}$. This assumption might not hold when singular systems of the form $E(x) \dot{x}=A(x) x$ are considered. For the case in which a degree dropping occurs, Theorem 2 could be extended using the results of Sideris and Barmish (1989) as well as Białas and Góra (2012).

Example 1. (Tanaka et al., 2003; González and Bernal, 2016) The nonlinear system

$$
\dot{x}=\left[\begin{array}{cc}
-3.5-1.5 a \sin x_{1} & -4 \\
9.5-10.5 b \sin x_{1} & -2
\end{array}\right] x
$$

can be rewritten as the convex sum $\dot{x}=\sum_{i=0}^{1} w_{i} A_{i} x$ with

$$
\begin{gathered}
A_{0}=\left[\begin{array}{cc}
-3.5-1.5 a & -4 \\
9.5-10 . b & -2
\end{array}\right], A_{1}=\left[\begin{array}{cc}
-3.5+1.5 a & -4 \\
9.5+10.5 b & -2
\end{array}\right] \\
w_{0}=\left(1-\sin \left(x_{1}\right)\right) / 2, \quad w_{1}=1-w_{0}
\end{gathered}
$$

Its stability properties with parameters $a \in[-4,4]$, $b \in[-1.5,1.5]$ can be analyzed via quadratic stability (Tanaka and Wang, 2001), yielding the feasibility set shown in Fig. 1 with the marks $(\square)$.

However, considering the generalized characteristic polynomial of (10) $p(s, x)=s^{2}+\left(1.5 a \sin x_{1}+5.5\right) s+$ $3 a \sin x_{1}-42 b \sin x_{1}+45$, which can be expressed as a convex combination $p(s, x)=\sum_{i=1}^{2} w_{i}(x) p_{i}(s)$, where $p_{1}(s)=s^{2}+(1.5 a+5.5) s+3 a-42 b+45$ and $p_{2}(s)=s^{2}+(-1.5 a+5.5) s-3 a+42 b+45$, conditions in Theorem 2 yield the feasibility set marked with $(x)$ in Fig. 1, it clearly overcomes the quadratic matrix-based analysis usually adopted in the quasi-LPV context, while preserving the LMI quality of solutions.

Example 2. Consider the uncertain nonlinear system

$$
\dot{x}=\left[\begin{array}{cc}
-5+\theta & x_{2} \\
x_{2} & -10+\theta+x_{2}
\end{array}\right] x
$$

within $\mathcal{C}=\left\{|\theta| \leq 2,\left|x_{2}\right| \leq 3\right\}$.

Its generalized characteristic polynomial is given by

$$
\begin{aligned}
p(s, x, \theta)= & s^{2}+\underbrace{\left(-2 \theta-x_{2}+15\right)}_{a_{1}(x, \theta)} s \\
& +\underbrace{\theta^{2}+\theta x_{2}-15 \theta-x_{2}^{2}-5 x_{2}+50}_{a_{0}(x, \theta)} .
\end{aligned}
$$

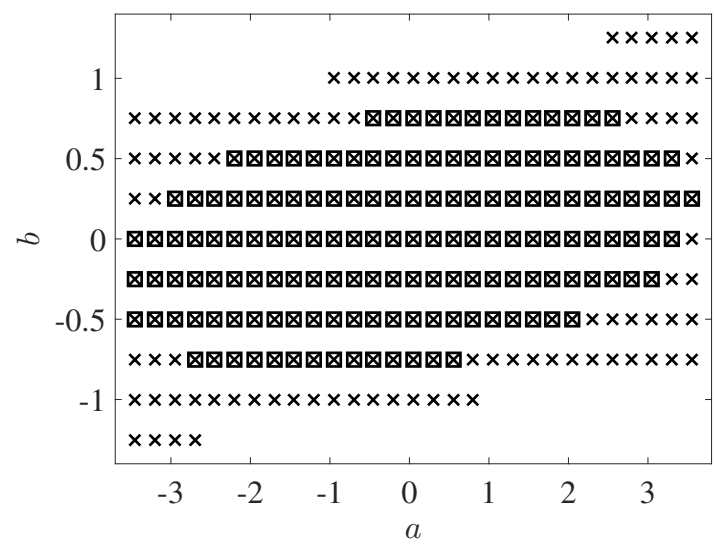

Fig. 1. Feasibility regions: quadratic approach $(\square)$ and polynomial convex approach $(\times)$.

In Fig. 2, the coefficient space of the previous polynomial is illustrated as black dots; it is obtained by considering the way $a_{1}$ and $a_{2}$ vary when the state $x_{2}$ and the uncertainty $\theta$ vary in $\mathcal{C}$.

A convex rewriting of (12) can be obtained by considering $z_{1}=x_{2}, z_{2}=x_{2}^{2}, \zeta_{1}=\theta$, and $\zeta_{2}=$ $\theta^{2}$; this choice is not unique and, as seen later, may be determinant in the feasibility of the proposed test, a characteristic which is shared by any polytope-based methodology (Kwiatkowski et al., 2006):

$$
p(s, x, \theta)=\sum_{i_{1}=0}^{1} \sum_{i_{2}=0}^{1} \sum_{j_{1}=0}^{1} \sum_{j_{2}=0}^{1} w_{i_{1}}^{1} w_{i_{2}}^{2} \omega_{j_{1}}^{1} \omega_{j_{2}}^{2} p_{i_{1} i_{2} j_{1} j_{2}}(s),
$$

with $w_{0}^{1}=\left(3-z_{1}\right) / 6, w_{1}^{1}=1-w_{0}^{1}, w_{0}^{2}=\left(9-z_{2}\right) / 9$, $w_{1}^{2}=1-w_{0}^{2}, \omega_{0}^{1}=\left(2-\zeta_{1}\right) / 4, \omega_{1}^{1}=1-\omega_{0}^{1}$, $\omega_{0}^{2}=\left(4-\zeta_{2}\right) / 4, \omega_{1}^{2}=1-\omega_{0}^{2}$, and 16 vertex polynomials $p_{i_{1} i_{2} j_{1} j_{2}}(s)=\left.p(s, x, \theta)\right|_{w_{i_{1}}^{1}=w_{i_{2}}^{2}=\omega_{j_{1}}^{1}=\omega_{j_{2}}^{2}=1}$, which constitute a box in the space $z-\zeta$. The coefficients of these polynomials are marked in Fig. 2 with empty circles along with their convex hull (the gray closed polyhedral including all of them); clearly, the convex hull of only 5 of these vertex polynomials enclose the same region as the one enclosed by the original 16 , i.e., 11 of them are redundant. The nonredundant vertex polynomials can be extracted by convex hull algorithms, e.g., quickhull (Barber et al., 1996); their use reduces the number of edges to be checked in Theorem 2 from $m=16 \times 15 / 2=$ 120 to only 5 . Thus, for 2 nd-order systems, the number of LMIs can be reduced from $2^{p+\rho-1}\left(2^{p+\rho}-1\right)$ to $2^{p+\rho}$ or fewer.

The product and powers of convex sums are convex sums themselves 4 thus, if $z_{1}=x_{2}$ and $\zeta_{1}=\theta$, then $x_{2}^{2}$ and $\theta^{2}$ can be written as nested convex sums of the former variables: $z_{2}=x_{2}^{2}=\left(w_{0}^{1}(-3)+w_{1}^{1}(3)\right)^{2}, \zeta_{2}=$

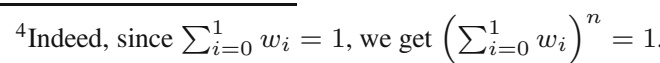




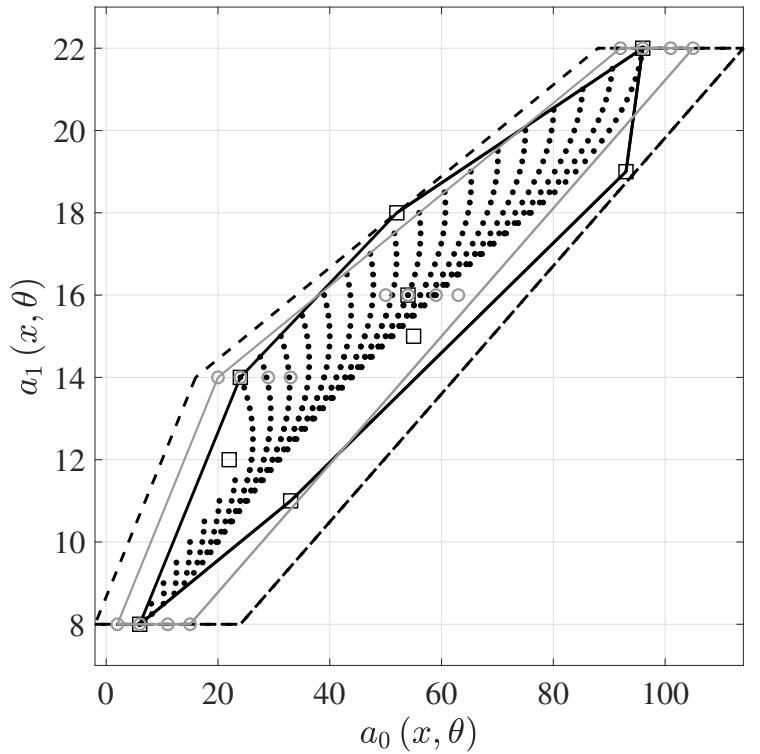

Fig. 2. Coefficient space of (12): (•), vertex polynomials of 13]: (०), convex hull of 13]: (-), "associated" vertex polynomials of (14): ( $\square$ ), convex hull of "associated" (14): (-), unstable result from (14): (- -).

$\left(\omega_{0}^{1}(-2)+\omega_{1}^{1}(2)\right)^{2}$. In this way, 12 can be rewritten without the need of introducing $w_{0}^{2}, w_{1}^{2}, \omega_{0}^{2}$, and $\omega_{1}^{2}$ :

$$
p(s, x, \theta)=\sum_{i_{1}=0}^{1} \sum_{i_{2}=0}^{1} \sum_{j_{1}=0}^{1} \sum_{j_{2}=0}^{1} w_{i_{1}}^{1} w_{i_{2}}^{1} \omega_{j_{1}}^{1} \omega_{j_{2}}^{1} p_{i_{1} i_{2} j_{1} j_{2}}(s) \text {. }
$$

If similar terms are not algebraically associated (those sharing the same $w-\omega$ decomposition), 16 vertex polynomials are again obtained; the corresponding convex hull is the polyhedral in dashed black lines shown in Fig. 2. it includes one unstable vertex which makes the stability of the system (11) impossible to establish via Theorem 2

On the other hand, if similar terms in (14) are associated, less conservative results arise as the convex sum passes from 16 to 9 different terms, which correspond to the vertexes shown in Fig. 2 with empty square marks; the corresponding convex hull is the polyhedral in solid black lines for which Theorem 2yields a feasible solution.

Whenever a nested convex sum appears in the polytopic representation (6), it can benefit from asymptotically exact sum relaxations based on the Pólya theorem (Sala and Ariño, 2007). This can be done in this example as (14) has double sums. Conditions thus obtained progressively enclose the actual coefficient space further reducing conservativeness.

\section{Robust control}

A direct way to employ the results of the previous section for stabilization purposes consists in placing poles (via LMIs, for instance) of the vertex polynomials of the open-loop system; then, using a convex sum of the stabilizing gains as a control law and verifying the stability of the whole convex sum of stabilized polynomials. To do so, a control law in the form of a parallel distributed compensation (PDC) (Baumann and Rugh, 1986; Wang et al., 1996) can be used, employing the same state-dependent weights $\mathbf{w}_{\mathbf{i}}$ used to convexly rewrite the generalised characteristic polynomial in (6):

$$
u(t)=F(x) x(t)=\sum_{\mathbf{v}} \mathbf{w}_{\mathbf{v}}(z(x)) F_{\mathbf{v}} x(t),
$$

with $F_{\mathbf{v}} \in \mathbb{R}^{m \times n}, \mathbf{v} \in\{0,1\}^{p}$, being gains stabilizing the uncertainty cluster $\sum_{\mathbf{i}} \sum_{\mathbf{j}} \mathbf{w}_{\mathbf{i}}(x) \omega_{\mathbf{j}}(x, \theta) p_{\mathbf{i j \mathbf { v }}}(s)$, which comes from the convex representation of the closed-loop generalized characteristic polynomial $p(s, x, \theta)=$ $\operatorname{det}(s I-A(x, \theta)-B(x, \theta) F(x))$ :

$$
p(s, x, \theta)=\sum_{\mathbf{i}} \sum_{\mathbf{j}} \sum_{\mathbf{v}} \mathbf{w}_{\mathbf{i}}(x) \mathbf{w}_{\mathbf{v}}(x) \omega_{\mathbf{j}}(x, \theta) p_{\mathbf{i j \mathbf { j }}}(s) .
$$

In other words, gains $F_{\mathbf{v}}$ should stabilize every uncertain variation of the corresponding vertex polynomial as uncertainty cannot be used for control purposes. This task can be performed by constructing as many Routh-Hurwitz tables as variations in $\mathbf{i}$ (states) and $\mathbf{j}$ (uncertainty) for each instance of $\mathbf{v}$ (gain index), a method resembling that employed by Barmish et al. (1992). The foregoing argumentations lead to simpler conditions for 2 nd-order systems since these polynomials are stable if and only if their coefficients are positive (Dorf and Bishop, 1998), something that can be straightforwardly tested via LMIs. The next example illustrates this discussion.

Example 3. Consider a slight variation of Example 1

$$
\dot{x}(t)=\left[\begin{array}{cc}
-3.5 & -4 \\
9.5+5.25 \sin x_{1} & -2
\end{array}\right] x(t)+\left[\begin{array}{l}
1 \\
0
\end{array}\right] u(t),
$$

with $u(x)=F(x) x=\left[\begin{array}{ll}f_{1}(x) & f_{2}(x)\end{array}\right] x$; the closed-loop system is

$$
\dot{x}(t)=\left[\begin{array}{cc}
-3.5+f_{1}(x) & -4+f_{2}(x) \\
9.5+21 \sin x_{1} & -2
\end{array}\right] x(t),
$$

and the generalized characteristic polynomial is given by

$$
\begin{aligned}
p(s, x)= & s^{2}+\left(5.5-f_{1}(x)\right) s \\
& +\sin x_{1}\left(84-21 f_{2}(x)\right) \\
& -9.5 f_{2}(x)-2 f_{1}(x)+45 .
\end{aligned}
$$

Using the convex modelling techniques of the previous section, this polynomial can be rewritten as

$$
\begin{aligned}
p(s, x)= & \sum_{i=0}^{1} w_{i}(x)\left\{s^{2}+\left(5.5-f_{1}(x)\right) s\right. \\
& +z_{i}\left(84-21 f_{2}(x)\right) \\
& \left.-9.5 f_{2}(x)-2 f_{1}(x)+45\right\},
\end{aligned}
$$


with $w_{0}=\left(1-\sin x_{1}\right) / 2, w_{1}=1-w_{0}, z_{0}=-1$, and $z_{1}=1$. Once the proposed PDC control structure $F(x)=$ $\sum_{v=0}^{1} w_{v}(x)\left[\begin{array}{ll}f_{1}^{v} & f_{2}^{v}\end{array}\right]$ is applied, the polynomial can be written as a double nested convex sum:

$$
\begin{aligned}
p(s, x)= & \sum_{i=0}^{1} \sum_{v=0}^{1} w_{i}(x) w_{v}(x)\left\{s^{2}+\left(5.5-f_{1}^{v}\right) s\right. \\
& \left.+z_{i}\left(84-21 f_{2}^{v}\right)-9.5 f_{2}^{v}-2 f_{1}^{v}+45\right\} .
\end{aligned}
$$

Due to the system order and the fact that the open-loop system consists only of two vertex polynomials, stabilization is reduced to finding a pair of gains $F_{0}=\left[\begin{array}{ll}f_{1}^{0} & f_{2}^{0}\end{array}\right]$ and $F_{1}=\left[\begin{array}{ll}f_{1}^{1} & f_{2}^{1}\end{array}\right]$ stabilizing the polynomials $p_{i v}(s)=s^{2}+\left(5.5-f_{1}^{v}\right) s+$ $z_{i}\left(84-21 f_{2}^{v}\right)-9.5 f_{2}^{v}-2 f_{1}^{v}+45, i, v=\{0,1\}$. These gains are the decision variables of the following LMIs, which ensure that the polynomials have positive coefficients:

$$
z_{i}\left(84-21 f_{2}^{v}\right)-9.5 f_{2}^{v}-2 f_{1}^{v}+45>0, \quad 5.5-f_{1}^{v}>0,
$$

for $i, v=\{0,1\}$. These LMI conditions can be relaxed by associating the polynomials $p_{i v}(s)$; a feasible solution is $f_{1}^{0}=-41.0133, f_{2}^{0}=1.8452, f_{1}^{1}=-41.0133$, and $f_{2}^{1}=1.8452$. In Fig. 3 it is shown how these gains stabilize the system along with the control law that they produce.
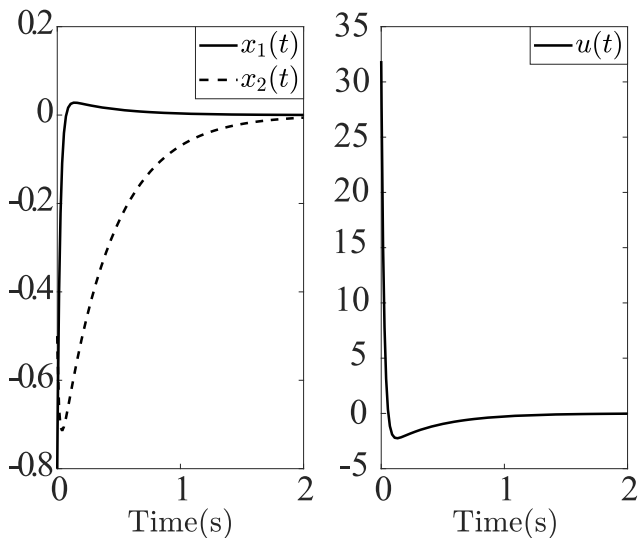

Fig. 3. Stabilised states $x(t)$ and control law $u(t)$.

Clearly, finding gains $F_{\mathbf{v}}$ for which some guarantee of feasibility exists is a hard task. In the context of single-input single-output (SISO) LPV systems, Henrion et al. (2003) considered robust stabilization of a polytope of polynomials with a fixed-order controller. Naturally, the conditions thereby proposed are also only sufficient: failing to find a feasible solution does not discard the existence of a controller. This methodology is now extended to our proposal while preserving their LMI nature; to do so, let $a_{\mathrm{ijv}}^{l}, l \in\{0,1, \ldots, n-$
$1\}, a_{\mathbf{i j v}}^{n}=1$, denote the coefficients of the vertex polynomials $p_{\mathbf{i j v}}$ in (16), grouped in a vector as $a_{\mathbf{i j v}}=\left[\begin{array}{lllll}a_{\mathbf{i j v}}^{0} & a_{\mathbf{i j v}}^{1} & \cdots & a_{\mathbf{i j v}}^{n-1} & 1\end{array}\right]$. Recall that these coefficients depend linearly on the entries of $F_{\mathbf{v}} \in \mathbb{R}^{1 \times n}$, where $F_{\mathbf{v}}=\left[\begin{array}{llll}f_{\mathbf{v}}^{1} & f_{\mathbf{v}}^{2} & \cdots & f_{\mathbf{v}}^{n}\end{array}\right]$. Consider also a given stable polynomial $d_{n} s^{n}+d_{n-1} s^{n-1}+\cdots+d_{1} s+d_{0}$, whose coefficients will be grouped in a vector $d=$ $\left[\begin{array}{lllll}d_{0} & d_{1} & \cdots & d_{n-1} & d_{n}\end{array}\right]$. Under these hypotheses, the following result is established.

Theorem 3. The origin of a SISO uncertain nonlinear system of the form (1), having a generalized characteristic polynomial (16) in the compact set $\mathcal{C}$ under the control law (15), is asymptotically stable if there are matrices $Q_{\mathbf{i j v}}=Q_{\mathbf{i j v}}^{T} \in \mathbb{R}^{n \times n}, \mathbf{i}, \mathbf{v} \in\{0,1\}^{p}, \mathbf{j} \in\{0,1\}^{\rho}$, and gain entries $f_{\mathrm{v}}^{l}, l \in\{1,2, \ldots, n\}$, such that the following LMIs are feasible:

$$
\begin{aligned}
& \sum_{k=1}^{n} \sum_{l=1}^{n}\left(\Pi_{k}^{T}\left[\begin{array}{ll}
0 & 1 \\
1 & 0
\end{array}\right] \Pi_{l}+\Pi_{l}^{T}\left[\begin{array}{ll}
0 & 1 \\
1 & 0
\end{array}\right] \Pi_{k}\right) Q_{\mathbf{i j v}}^{k l} \\
& \quad+a_{\mathbf{i j v}}^{T} d+d^{T} a_{\mathbf{i j v}}-2 \gamma d^{T} d \geq 0
\end{aligned}
$$

for an arbitrarily small $\gamma>0$, with $\Pi_{i} \in \mathbb{R}^{2 \times(n+1)}$ being matrices with ones at entries $(1, i)$ and $(2, i+1)$, and zeros elsewhere.

Proof. Using convex sum properties on 18, i.e., taking into account that $\sum_{\mathbf{i}} \mathbf{w}_{\mathbf{i}}=1,0 \leq \mathbf{w}_{\mathbf{i}} \leq 1, \sum_{\mathbf{j}} \omega_{\mathbf{j}}=1$, $0 \leq \omega_{\mathbf{j}} \leq 1$, and omitting arguments when convenient, yields

$$
\begin{aligned}
& \sum_{\mathbf{i}} \sum_{\mathbf{j}} \sum_{\mathbf{v}} \mathbf{w}_{\mathbf{i}}(x) \mathbf{w}_{\mathbf{v}}(x) \omega_{\mathbf{j}}(x, \theta)\left\{a_{\mathbf{i j \mathbf { v }}}^{T} d+d^{T} a_{\mathbf{i j \mathbf { j }}}-2 \gamma d^{T} d\right. \\
& \left.+\sum_{k=1}^{n} \sum_{l=1}^{n}\left(\Pi_{k}^{T}\left[\begin{array}{ll}
0 & 1 \\
1 & 0
\end{array}\right] \Pi_{l}+\Pi_{l}^{T}\left[\begin{array}{ll}
0 & 1 \\
1 & 0
\end{array}\right] \Pi_{k}\right) Q_{\mathbf{i j \mathbf { v }}}^{k l}\right\} \\
& =\left(\sum_{\mathbf{i}, \mathbf{j}, \mathbf{v}} \mathbf{w}_{\mathbf{i}} \mathbf{w}_{\mathbf{v}} \omega_{\mathbf{j}} a_{\mathbf{i j \mathbf { v }}}\right)^{T} d+d^{T} \underbrace{\left(\sum_{\mathbf{i}, \mathbf{j}, \mathbf{v}} \mathbf{w}_{\mathbf{i}} \mathbf{w}_{\mathbf{v}} \omega_{\mathbf{j}} a_{\mathbf{i j \mathbf { v }}}\right)}_{c}-2 \gamma d^{T} d \\
& +\sum_{k=1}^{n} \sum_{l=1}^{n}\left(\Pi_{k}^{T}\left[\begin{array}{ll}
0 & 1 \\
1 & 0
\end{array}\right]_{l}+\Pi_{l}^{T}\left[\begin{array}{ll}
0 & 1 \\
1 & 0
\end{array}\right] \Pi_{k}\right) \underbrace{\sum_{\mathbf{i}} \mathbf{w}_{\mathbf{v}} \omega_{\mathbf{j}} Q_{\mathbf{i j \mathbf { v }}}^{k l}}_{\mathbf{i}, \mathbf{j}, \mathbf{v}} \geq 0,
\end{aligned}
$$

which, by Theorem 1 by Henrion et al. (2003), allows establishing the desired result with $c$ and $Q$ as defined above with underbraces.

The result above can also be established using Theorem 2 by Henrion et al. (2003), which is concerned with robust stability of a convex sum of $N$ plants $b^{i}(s) / a^{i}(s)$ of order $n$ in a closed-loop configuration with a fixed $m$-th order controller of the form $y(s) / x(s)$; thereby, the closed-loop polynomials are denoted as 
$c^{i}(s)=a^{i}(s) x(s)+b^{i}(s) y(s)$ with coefficients grouped in vectors of the form $c^{i}=\left[\begin{array}{llll}c_{0}^{i} & c_{1}^{i} & \cdots & c_{m+n}^{i}\end{array}\right]$. Correspondingly, in our case the closed-loop polynomials are $p_{\mathbf{i j v}}$ with coefficients grouped in vectors $a_{\mathbf{i j v}}=$ $\left[\begin{array}{lllll}a_{\mathbf{i j v}}^{0} & a_{\mathbf{i j v}}^{1} & \cdots & a_{\mathbf{i j v}}^{n-1} & 1\end{array}\right]$.

Note that the PDC control law (15) in Theorem 3 includes that from the work of Henrion et al. (2003) as a particular case $\left(F_{\mathbf{v}}=F, \forall \mathbf{v}\right)$; it is a convex sum of order- 0 controllers (i.e., $m=0$ ), while the index triplet $(\mathbf{i}, \mathbf{j}, \mathbf{v})$ leads to $N=2^{(2 p+\rho)}$ LMIs of the form (18). The results just presented can be improved in two ways: exploiting Pólya-like relaxations on the double convex sums in (18) and replacing the stable polynomial $d(s)$ by a stable polytope of polynomials sharing the same weighting functions of the generalized characteristic polynomial.

Example 4. (Rhee and Won, 2006) We now turn our attention to a more complex system whose feasibility under different control laws has been examined in previous works (González et al., 2017; Pan et al., 2012):

$$
\begin{aligned}
\dot{x}(t)= & {\left[\begin{array}{cc}
0.5 a \sin x_{1}+0.5 a-\sin x_{1}+1 & 2.5 \sin x_{1}-7.5 \\
1.5-0.5 \sin x_{1} & \sin x_{1}+1
\end{array}\right] x(t) } \\
& +\left[\begin{array}{c}
0.5 b \sin x_{1}+0.5-0.5 \sin x_{1}+0.5 \\
0.5 \sin x_{1}+1.5
\end{array}\right] u(t) .
\end{aligned}
$$

A PDC controller has been fetched through four different approaches for each integer instance $(a, b)$ in $a \in[7,11]$ and $b \in[0,20]$; whenever a solution was found, a mark was put in the corresponding coordinate of Fig. 4. $(\times)$ when the LMI (17) was used, $(*)$ when Theorem 3 was employed without grouping polynomials sharing the weighting functions, ( $\square$ ) when both the LMI 17) and Theorem 3 were used with grouped polynomials, $(\circ)$ when Theorem 1 of González et al. (2017) was used (which is a nonquadratic proposal). As can be seen, the proposed methodology has increased the feasibility set of former approaches.

\section{Conclusion}

A methodology for stability and stabilization of uncertain nonlinear systems via an exact convex representation of their generalized characteristic polynomial was presented. Uncertainties and nonlinearities were exactly modeled within a polytope of polynomials allowing a richer class of controllers to take advantage of the available states while robustly stabilizing the plant. In contrast to its customary application to quasi-LPV models, convexity was proved to be advantageous when applied to the notion of a generalized characteristic polynomial; its LMI treatment and Pólya-like relaxations were preserved. Higher-order controllers (as those in the work of Henrion et al. (2003)), corresponding to dynamic control schemes, may better fit a polynomial approach as the one proposed in this work;

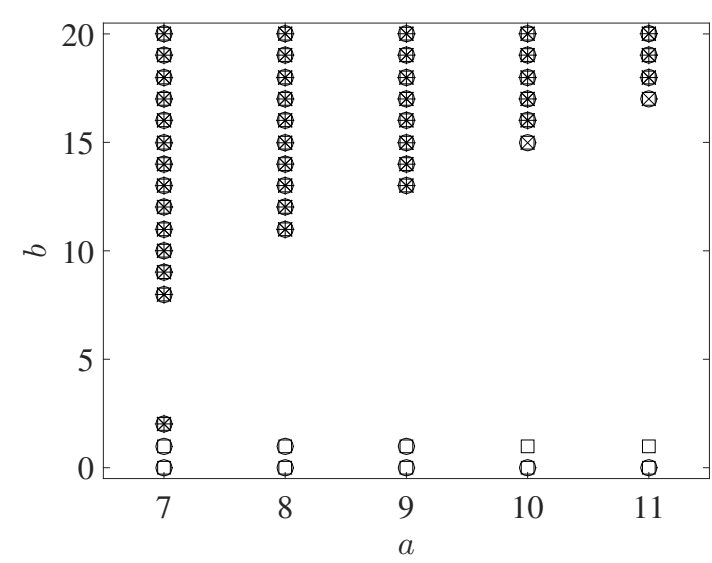

Fig. 4. Feasibility sets: LMI 17 without grouping polynomials $(\times)$, Theorem 3 without grouping polynomials $(*)$, LMI (17) and Theorem 3 (both with grouped polynomials) ( $\square$ ), Theorem 1 by González et al. (2017) (०).

as they might be relevant for observer-controller setups, future work may focus on these issues.

\section{Acknowledgment}

This work has been supported by the CONACYT scholarship 590759, the ITSON project PROFAPI 2017-0088, and the PFCE 2016-17.

\section{References}

Amato, F., Pironti, A. and Scala, S. (1996). Necessary and sufficient conditions for quadratic stability and stabilizability of uncertain linear time-varying systems, IEEE Transactions on Automatic Control 41(1): 125-128.

Apkarian, P. and Gahinet, P. (1995). A convex characterization of gain-scheduled $\mathrm{h}_{\infty}$ controllers, IEEE Transactions on Automatic Control 40(5): 853-864.

Barber, C.B., Dobkin, D.P. and Huhdanpaa, H. (1996). The quickhull algorithm for convex hulls, ACM Transactions on Mathematical Software (TOMS) 22(4): 469-483.

Barmish, B.R., Hollot, C.V., Kraus, F.J. and Tempo, R. (1992). Extreme point results for robust stabilization of interval plants with first-order compensators, IEEE Transactions on Automatic Control 37(6): 707-714.

Bartlett, A.C., Hollot, C.V. and Lin, H. (1988). Root locations of an entire polytope of polynomials: It suffices to check the edges, Mathematics of Control, Signals and Systems 1(1): 61-71.

Baumann, W. and Rugh, W. (1986). Feedback control of nonlinear systems by extended linearization, IEEE Transactions on Automatic Control 31(1): 40-46.

Białas, S. (1985). A necessary and sufficient condition for the stability of convex combinations of stable polynomials or matrices, Bulletin of the Polish Academy of Sciences 33(9-10): 473-480. 
Białas, S. and Góra, M. (2012). A few results concerning the Hurwitz stability of polytopes of complex polynomials, Linear Algebra and Its Applications 436(5): 1177-1188.

Boyd, S., Ghaoui, L.E., Feron, E. and Belakrishnan, V. (1994). Linear Matrix Inequalities in System and Control Theory, SIAM, Philadelphia, PA.

Dorf, R. and Bishop, R. (1998). Modern Control Systems, Pearson (Addison-Wesley), Upper Saddle River, NJ.

Ebihara, Y., Peaucelle, D., Arzelier, D., Hagiwara, T. and Oishi, Y. (2012). Dual LMI approach to linear positive system analysis, 12th International Conference on Control, Automation and Systems (ICCAS), JeJu Island, South Korea, pp. 887-891.

Gahinet, P., Nemirovski, A., Laub, A.J. and Chilali, M. (1995). LMI Control Toolbox, Math Works, Natick, MA.

González, T. and Bernal, M. (2016). Progressively better estimates of the domain of attraction for nonlinear systems via piecewise Takagi-Sugeno models: Stability and stabilization issues, Fuzzy Sets and Systems 297: 73-95.

González, T., Bernal, M., Sala, A. and Aguiar, B. (2017). Cancellation-based nonquadratic controller design for nonlinear systems via Takagi-Sugeno models, IEEE Transactions on Cybernetics 47(9): 2628-2638.

Guerra, T.M., Estrada-Manzo, V. and Lendek, Z. (2015). Observer design of nonlinear descriptor systems: An LMI approach, Automatica 52: 154-159.

Guerra, T.M. and Vermeiren, L. (2004). LMI-based relaxed non-quadratic stabilization conditions for nonlinear systems in Takagi-Sugeno's form, Automatica 40(5): 823-829.

Henrion, D., Sebek, M. and Kucera, V. (2003). Positive polynomials and robust stabilization with fixed-order controllers, IEEE Transactions on Automatic Control 48(7): 1178-1186.

Johansen, T.A. (2000). Computation of Lyapunov functions for smooth nonlinear systems using convex optimization, $A u$ tomatica 36(11): 1617-1626.

Khalil, H. (2002). Nonlinear Systems, 3rd Edn., Prentice Hall, Upper Saddle River, NJ.

Kharitonov, V. (1978). Asymptotic stability of an equilibrium position of a family of systems of linear differential equations, Differential Equations 14(11): 1483-1485.

Kwiatkowski, A., Boll, M. and Werner, H. (2006). Automated generation and assessment of affine LPV models, 45th IEEE Conference on Decision and Control, San Diego, CA, USA, pp. 6690-6695.

Pan, J., Guerra, T., Fei, S. and Jaadari, A. (2012). Nonquadratic stabilization of continuous T-S fuzzy models: LMI solution for a local approach, IEEE Transactions on Fuzzy Systems 20(3): 594-602.

Rhee, B. and Won, S. (2006). A new fuzzy Lyapunov function approach for a Takagi-Sugeno fuzzy control system design, Fuzzy Sets and Systems 157(9): 1211-1228.
Robles, R., Sala, A., Bernal, M. and González, T. (2017). Subspace-based Takagi-Sugeno modeling for improved LMI performance, IEEE Transactions on Fuzzy Systems 25(4): 754-767.

Sala, A. and Ario, C. (2009). Polynomial fuzzy models for nonlinear control: A Taylor series approach, IEEE Transactions on Fuzzy Systems 17(6): 1284-1295.

Sala, A. and Ariño, C. (2007). Asymptotically necessary and sufficient conditions for stability and performance in fuzzy control: Applications of Polya's theorem, Fuzzy Sets and Systems 158(24): 2671-2686.

Sanchez, M. and Bernal, M. (2017). A convex approach for reducing conservativeness of Kharitonov's-based robustness analysis, 20th IFAC World Congress, Toulouse, France, pp. 855-860.

Shamma, J.S. and Cloutier, J.R. (1992). A linear parameter varying approach to gain scheduled missile autopilot design, American Control Conference, Chicago, IL, USA, pp. 1317-1321.

Sideris, A. and Barmish, B.R. (1989). An edge theorem for polytopes of polynomials which can drop in degree, Systems \& Control Letters 13(3): 233-238.

Sturm, J.F. (1999). Using SeDuMi 1.02, a MATLAB toolbox for optimization over symmetric cones, Optimization Methods and Software 11(1-4): 625-653.

Tanaka, K., Hori, T. and Wang, H. (2003). A multiple Lyapunov function approach to stabilization of fuzzy control systems, IEEE Transactions on Fuzzy Systems 11(4): 582-589.

Tanaka, K. and Wang, H. (2001). Fuzzy Control Systems Design and Analysis. A Linear Matrix Inequality Approach, John Wiley \& Sons, New York, NY.

Taniguchi, T., Tanaka, K. and Wang, H. (2001). Model construction, rule reduction and robust compensation for generalized form of Takagi-Sugeno fuzzy systems, IEEE Transactions on Fuzzy Systems 9(2): 525-537.

Tapia, A., Bernal, M. and Fridman, L. (2017). Nonlinear sliding mode control design: An LMI approach, Systems \& Control Letters 104: 38-44.

Wang, H., Tanaka, K. and Griffin, M. (1996). An approach to fuzzy control of nonlinear systems: Stability and design issues, IEEE Transactions on Fuzzy Systems 4(1): 14-23.

Xu, S., Darouach, M. and Schaefers, J. (1993). Expansion of $\operatorname{det}(a+b)$ and robustness analysis of uncertain state space systems, IEEE Transactions on Automatic Control 38(11): 1671-1675.

Marcelino Sánchez was born in Los Mochis, Mexico, in 1992. He obtained his BSc degree in mechatronics engineering and his MSc degree in control systems at the Sonora Institute of Technology, Mexico, in 2015 and 2017, respectively. He is currently pursuing a $\mathrm{PhD}$ degree at the Polytechnic University of Hauts-de-France. His research interests include the analysis and synthesis of nonlinear control systems based on convex structures and linear matrix inequalities, and the synthesis of control algorithms for energy management in hybrid vehicles. 
Miguel Bernal was born in Guadalajara, Mexico, in 1976. He received his PhD degree in automatic control from Czech Technical University in Prague in 2005. He was a post-doctoral researcher at the University of Valenciennes and Hainaut-Cambrésis, France, from 2006 to 2009. He has been a member of the National Research System of Mexico since 2007. In 2011 he was appointed a full professor at the Sonora Institute of Technology, Mexico, where he is the head of postgraduate programs in control and systems. He currently leads and supervises several research projects and $\mathrm{PhD}$ theses in Mexico and abroad on nonlinear control via convex structures and linear matrix inequalities, an area where he counts more than 30 journal papers and over 1000 citations.

Received: 13 July 2018

Revised: 21 December 2018

Accepted: 21 January 2019 